\title{
RG decimation study of SU(2) gauge theory
}

\author{
E.T. Tomboulis and Alexander Velytsky* \\ Department of Physics and Astronomy, UCLA, Los Angeles, CA 90095-1547, USA \\ E-mail: tombouli@physics.ucla.edu, velephysics.ucla.edu
}

\begin{abstract}
We report on numerical studies of RG decimations in SU(2) gauge theory. We study in particular a class of plaquette actions involving sums of group representations. We measure a number of observables representative of different length scales in order to investigate the transformation of the system under different choices of spin blocking, and examine the flow of the effective action couplings. A need for a projection to some class of effective actions on the configurations resulting from the adopted numerical decimation procedures is demonstrated. A numerical decimation procedure resulting in an effective single plaquette Lagrangian tailored to reproduce different medium/large scale physics is devised.
\end{abstract}

XXIV International Symposium on Lattice Field Theory

July 23-28 2006

Tucson Arizona, US

${ }^{*}$ Speaker. 


\section{Introduction}

The Monte Carlo Renormalization Group (MCRG) technique is a useful tool for constructing an improved action with reduced discretization errors on the lattice. It has been extensively used in the search for the 'perfect action' [1], for which, under blocking transformations, trajectory flows approach the Wilsonian 'renormalized trajectory', and lattice artifacts disappear.

Under RG evolution any starting action generally develops a variety of additional couplings. An adequate model of the resulting effective action must, therefore, include a choice of several such couplings. In the past effective models with actions consisting of various closed loops in the fundamental representation [2], or a mixed fundamental-adjoint single plaquette action [3] have been studied. Systematic errors due to the truncation of the phase space, however, can be significant and prevent the effective model from reaching the renormalized trajectory. Also it is a priori unclear if a configuration obtained after the numerical block spinning is representative of the equilibrium configurations of the adopted effective model.

After one or more block spinnings are performed, starting from a simple (e.g. Wilson) action, one needs to measure the set of couplings retained in one's model of the effective action. This may be achieved by use of demon [4] or Schwinger-Dyson methods [2].

Our goal is to study the long distance confining regime of non-Abelian gauge theory. Various RG decimation schemes can be employed in order to connect perturbative short-scale with nonperturbative long scale physics. In Ref. [5] an exact analytical decimating procedure was devised which imposes lower and upper bounds on such quantities as the partition function, and the partition function in the presence of a vortex, and other related quantities. The procedure can also be implemented numerically. Other numerical decimation schemes have been proposed before and are explored below.

We report some progress on the construction of an effective single plaquette action model which is designed to reproduce long/medium scale physics correctly. For this we devise special decimating procedures, which target specific medium and large distance observables (Wilson loops, static quark potential etc.). We pursue both analytical and numerical procedures. Here, we report mainly on a numerical decimation study.

\section{Decimation procedures}

Starting with a reflection positive plaquette action, e.g. the fundamental representation Wilson action, on lattice $\Lambda$ of spacing $a$, after $n$ decimation steps $(a \rightarrow \lambda a)$ the partition function is

$$
Z_{\Lambda}(\beta, n)=\exp \left(\sum_{m=0}^{n} \phi^{(m)}|\Lambda| / \lambda^{m d}\right) Z_{\Lambda^{(n)}}
$$

where $\phi^{(m)}$ denotes the bulk free energy generated by each $\lambda^{(m-1)} a \rightarrow \lambda^{(m)} a$ step, and $Z_{\Lambda^{(n)}}$ is the resulting partition function at scale $\lambda^{n} a$. For a class of decimations of the 'potential moving' type characterized by certain parameters it was shown in [5] that: (a) the action in $Z_{\Lambda^{(n)}}$ retains one-plaquette form, but contains generally all representations; (b) there is a range of values of the parameters for which each decimation step results in an upper bound, $Z_{\Lambda}(\beta, n-1) \leq Z_{\Lambda}(\beta, n)$; (c) there is another range of parameter values for which each decimation step results in a lower 
bound, $Z_{\Lambda}(\beta, n-1) \geq Z_{\Lambda}(\beta, n)$. It is then possible to introduce a single parameter $\alpha$ which, at each decimation step, interpolates between the upper and lower bound, and hence has a value that keeps the partition function constant, i.e. exact under each successive step.

The same development can be generalized to apply in presence of other observables, in particular vortex free energies, i.e. twisted partition functions. Other quantities of interest, such as Wilson loops, Polyakov loops and 't Hooft loops, can be related to the vortex free energy through known inequalities. This procedure then leads to exact analytical results, and can also be applied as a numerical RG procedure. Its numerical implementation is under development.

Here we report on numerical decimations utilizing two other well-known available numerical procedures. For blocking by a factor 2 in all lattice directions $(a \rightarrow 2 a)$, which we use throughout in this study, these are:

- Swendsen decimation [6]

$$
Q_{\mu}(n)=U_{\mu}(n) U_{\mu}(n+\hat{\mu})+c \sum_{v \neq \mu} U_{v}(n) U_{\mu}(n+\hat{v}) U_{\mu}(n+\hat{v}+\hat{\mu}) U_{-v}(n+\hat{v}+2 \hat{\mu})
$$

- Double Smeared Blocking [7]

$$
\begin{aligned}
& U_{\mu}(n)=(1-6 c) U_{\mu}(n)+c \sum_{v \neq \mu} U_{v}(n) U_{\mu}(n+\hat{v}) U_{v}^{\dagger}(n+\hat{\mu}) \quad \times 2 \text { times } \\
& Q_{\mu}(n)=U_{\mu}(n) U_{\mu}(n+\hat{\mu}) .
\end{aligned}
$$

Here $c$ is the parameter which controls the relative weight of staples. For the Swendsen decimation $c=0.5$ and 1 values have been used. For the double smeared blocking, the classical limit value $c=0.077$ has been used [8].

For our numerical decimation, we choose to start from the standard Wilson action. After a decimated configuration is obtained, we need to 'project' it to some effective action. Motivated by the exact decimation procedure, we assume that a single plaquette action

$$
S=\sum_{j=1 / 2}^{N_{r}} \beta_{j}\left[1-\frac{1}{d_{j}} \chi_{j}\left(U_{p}\right)\right],
$$

truncated at some high representation $N_{r}$, is a general form of the effective action.

It is important to note that the decimated configurations may not represent equilibrium configurations of a particular effective action. Therefore we follow the microcanonical [ $₫$ ] evolution of the effective model starting from the decimated configurations.

\subsection{Numerical methods}

To compare the effective model to the decimated model, we need an efficient way to simulate a gauge theory with action (2.4). We use a procedure due to Hasenbusch and Necco [9]. The fundamental representation part of the action with specially tuned coupling is used to generate trial matrices for the metropolis updating. This procedure typically achieves $80 \%$ acceptance rate for the metropolis algorithm at the used couplings. Alternatively one could use a newly developed biased metropolis algorithm [10]. Simple heatbath updating is used only in the case of purely fundamental representation action. 
For the microcanonical updating and demon measurements we implement an improved algorithm, which retains demon energy values [11], making the demon canonical. The demons energies are restricted to $\left[-E_{\max }, E_{\max }\right]$, thus preventing demons from 'running away' with all the energy. The couplings of the effective action can be obtained as solutions of the equation

$$
\left\langle E_{d}\right\rangle=1 / \beta-E_{\max } / \tanh \left[\beta E_{\max }\right] .
$$

In table (1) we demonstrate the ability of the canonical demon method in measuring the couplings on $8^{4}$ lattice. An ensemble of 3000 configurations with couplings listed in the first row of the table is used. Demon is allowed 1 sweep for reaching equilibrium, than 10 sweeps for measurements. The measured couplings are listed on the second row of the table and are in good agreement with the initial values.

\begin{tabular}{|c|c|c|c|c|c|}
\hline & $\beta_{1 / 2}$ & $\beta_{1}$ & $\beta_{3 / 2}$ & $\beta_{2}$ & $\beta_{5 / 2}$ \\
\hline in & 2.2578 & -0.2201 & 0.0898 & -0.0333 & 0.0125 \\
\hline demon & $2.2580(4)$ & $-0.2206(4)$ & $0.0903(5)$ & $-0.0336(5)$ & $0.0127(4)$ \\
\hline
\end{tabular}

Table 1: Measurements of couplings by canonical demon method.

\section{Decimation study}

We fix the effective action to have 8 consecutive representations, starting from the fundamental. A $32^{4}$ lattice at $\beta=2.5$ is decimated once, using Swendsen type decimation with various staple weights $c$. In Fig. 1 (left) we show the fundamental representation demon energy flow, starting from $c=0.1$ Swendsen decimated configurations. We note that there is a significant demon
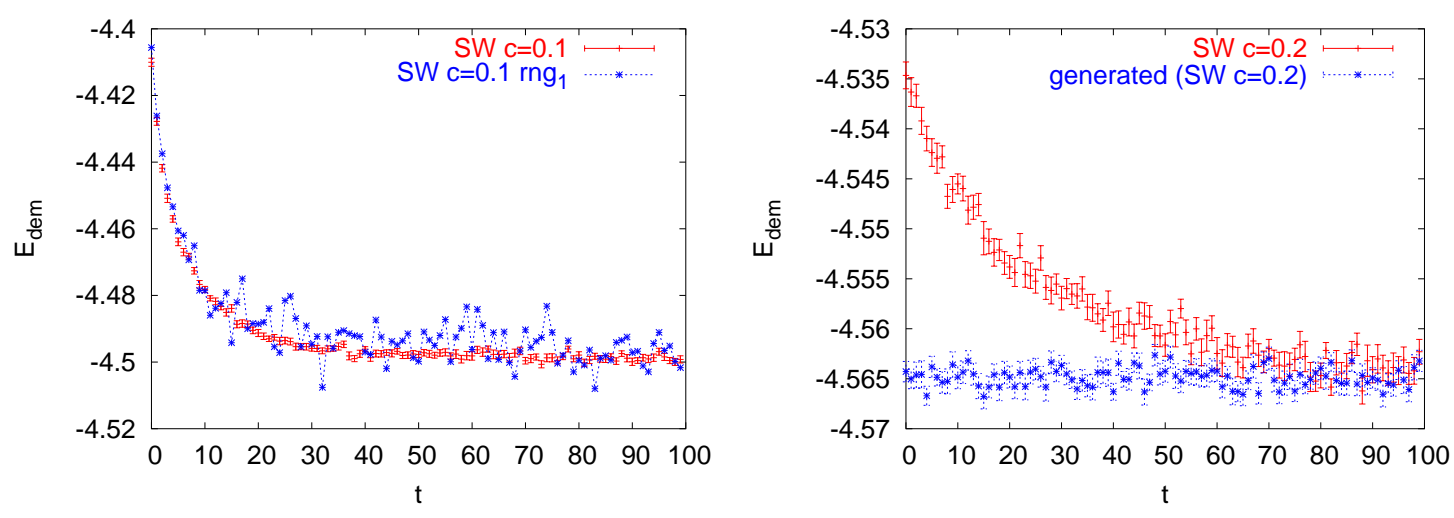

Figure 1: Demon fundamental energy flow for $c=0.1$ Swendsen decimated configurations. The average and a single demon run (left). Demon fundamental energy flow for $c=0.2$ Swendsen decimated configurations and for configurations generated with an effective action (right).

energy change during the microcanonical evolution. The change for different replicas is always in the same direction. There is a noticeable trend for flow stabilization at $\sim 100$ sweeps.

Next we let the demon reach equilibrium ( $>100$ sweeps) and then measure the couplings of the effective action (2.4). We then simulate this model and generate thermalized configurations. We 


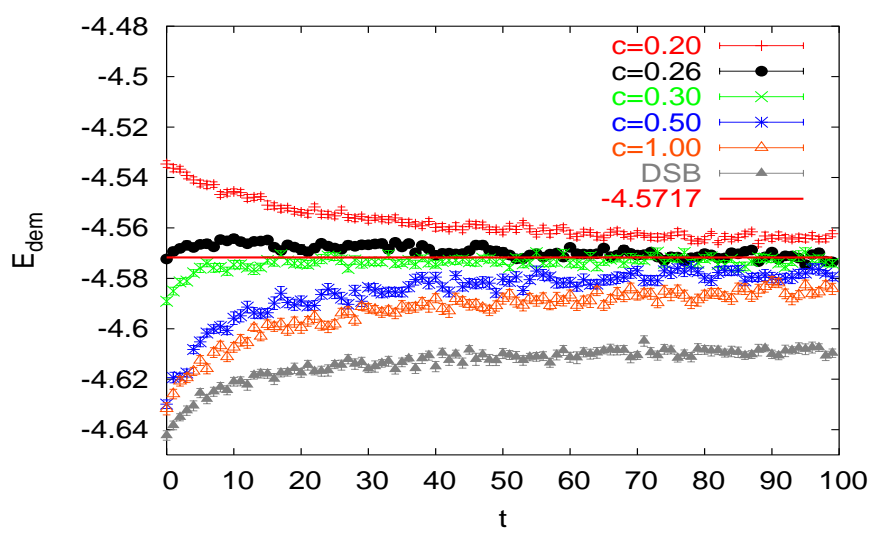

Figure 2: Demon fundamental energy flow for various decimated configurations (Swendsen and Double Smeared Blocking).

then compare the demon evolution on these thermalized configurations with the demon evolution on the $c=0.2$ Swendsen decimated configurations (Fig. 1 (right)). We see that in the former case there is no change in the demon energy, which indicates a very fast demon equilibration. Whereas in the latter case there is a pronounced change - this pronounced energy change is clearly due to configuration equilibration during the microcanonical evolution.

Therefore one concludes that starting from the (Swendsen) decimated configurations sufficient microcanonical evolution has to occur in order to 'project' into the equilibrium configurations of the effective model.

Next we vary the staple weight parameter $c$ and observe the demon energy flow. In Fig. 目 we demonstrate the fundamental demon energy evolution for $c=0.2, \ldots, 1.0$ Swendsen decimations and for the double smeared blocking with the classical $c$ value. We observe that there is a special $c \approx 0.26$ value, when right from the start there is little demon energy change. These particular decimation configurations are very close to the equilibrium configuration of the model (2.4). In Fig. 3 we look at the adjoint demon energy flow. We notice that there is a small change for $c=0.26$, while for $c=0.3$ it stays constant. This indicates that for the truncated actions the decimation can produce configurations which are only approximately in equilibrium and the projection is generally needed.

Next we compare some medium scale physical observables measured on the decimated configurations and on configurations obtained from the effective models. First for the $c=0.2$ Swendsen decimation we check the difference between $N \times N$ Wilson loops measured on the decimated configuration immediately after the decimation and after the projection of 100 sweeps, see Tab. 2. We see that it is indeed the process of 'projection' that makes the difference in observables decrease.

As the next step we would like to fix the decimation parameter at the value which minimizes the difference at the largest Wilson loop that we measure. In Tab. 3 we present the results for Swendsen type decimation and Wilson loops up to $3 \times 3$. We note that for different scale observables the smallest difference occurs at different $c$ values. It is interesting that for the largest Wilson loop the best results are obtained for $c=0.26$ - the value which produces configurations closest to equilibrium. 


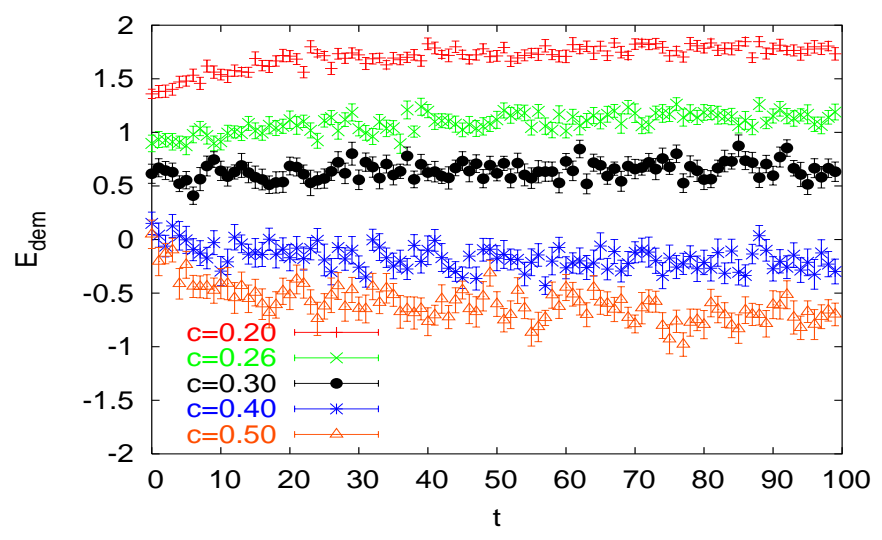

Figure 3: Demon adjoint energy flow for various Swendsen decimated configurations.

\begin{tabular}{|c||c|c|c|c|}
\hline$e q / m$ & $\beta_{1 / 2}, \beta_{1}, \beta_{3 / 2}, \ldots$ & $\Delta P / P^{\text {dec }}$ & $\Delta W_{2 \times 2} / W_{2 \times 2}^{\text {dec }}$ & $\Delta W_{3 \times 3} / W_{3 \times 3}^{\text {dec }}$ \\
\hline \hline $0 / 1$ & $2.1391(5),-0.1628(9), 0.0637(11)$, & & & \\
& $-0.0250(1), 0.0098(15)$ & $-0.0642(1)$ & $-0.2832(5)$ & $-0.7196(9)$ \\
\hline \multirow{2}{*}{$100 / 20$} & $2.2963(4),-0.2351(5), 0.0955(7)$, & & & \\
& $-0.0357(9), 0.0131(11),-0.0050(12)$ & $-0.0045(1)$ & $-0.0296(10)$ & $-0.3912(20)$ \\
\hline
\end{tabular}

Table 2: Canonical demon measured couplings after the $c=0.2$ Swendsen decimation and difference of various size Wilson loops estimates measured on decimated versus effective action configurations. Measurements performed right after the decimation and after 100 sweeps (measurements 20 sweeps).

The classical $c$ value of double smeared blocking produces results which are incapable of reproducing large scale physics correctly. There is obviously a need also in this case for a procedure similar to that described here for Swendsen type decimation.

\section{Summary}

Multirepresentation-single plaquette actions can effectively reproduce long-scale physics. We demonstrated that a procedure naturally leading to a projection to some class of effective actions is needed on the configurations resulting from any particular adopted numerical decimation scheme. Such numerical decimation procedures applied to the effective single plaquette Lagrangian can be tailored to reproduce different medium/large scale physics.

It is possible to extend the study by looking at the inter-quark potential. This would allow one to probe all length scales and check the effective action model and decimated configurations correspondence. There is also a possibility to compare numerical and exact decimation procedures. Numerically obtained coupling values can be used to connect to the exact decimation and for consistency checks.

\section{Acknowledgments}

We thank Academical Technology Services at UCLA for computer support. The simulations were performed on a PC cluster at UCLA. This work was in part supported by NSF-PHY-0309362. 


\begin{tabular}{|c|c|c|c|c|}
\hline$c$ & $\beta_{1 / 2}, \beta_{1}, \beta_{3 / 2}, \ldots$ & $\Delta P / P^{d e c}$ & $\Delta W_{2 \times 2} / W_{2 \times 2}^{d e c}$ & $\Delta W_{3 \times 3} / W_{3 \times 3}^{\text {dec }}$ \\
\hline 0.1 & $\begin{array}{l}1.9912(3),-0.3085(4), \\
0.0990(4),-0.0362(6), \\
0.0139(7),-0.0045(8)\end{array}$ & $-0.0001(1)$ & $-0.4160(6)$ & $-0.8899(11)$ \\
\hline 0.2 & $\begin{array}{c}2.2963(4),-0.2351(5) \\
0.0955(7),-0.0357(9) \\
0.0131(11),-0.0050(12)\end{array}$ & $-0.0045(1)$ & $-0.0296(10)$ & $-0.3912(20)$ \\
\hline 0.26 & $\begin{array}{c}2.3351(7),-0.1449(10), \\
0.0766(12),-0.0279(13), \\
0.0084(17)\end{array}$ & $-0.0038(12)$ & $0.1502(11)$ & $0.0926(29)$ \\
\hline 0.3 & $\begin{array}{c}2.3447(8),-0.0869(12) \\
0.0628(14),-0.0236(15) \\
0.0075(20)\end{array}$ & $-0.0006(1)$ & $0.2545(12)$ & $0.4559(41)$ \\
\hline 0.5 & $\begin{array}{c}2.3618(9), 0.0866(13), \\
0.0070(17),-0.0027(20), \\
-0.0013(22)\end{array}$ & $0.0082(11)$ & $0.4780(14)$ & $1.5029(69)$ \\
\hline 1.0 & $\begin{array}{c}2.4033(9), 0.1150(14), \\
-0.0274(18), 0.0071(22), \\
-0.0041(29)\end{array}$ & $0.0092(1)$ & $0.4456(14)$ & $1.4845(75)$ \\
\hline$\overline{\mathrm{DS}}$ & $\begin{array}{c}2.5463(11),-0.1167(17), \\
0.0320(23),-0.0055(28)\end{array}$ & $0.0068(1)$ & $0.4149(14)$ & $1.2697(70)$ \\
\hline
\end{tabular}

Table 3: Canonical demon measured couplings after different decimations and difference of various size Wilson loops estimates measured on decimated and generated with effective action configurations. Thermalization 100 sweeps, measurements 20 sweeps.

\section{References}

[1] P. Hasenfratz, Nucl. Phys. Proc. Suppl. 63, 53 (1998) [arXiv:hep-lat/9709110].

[2] P. de Forcrand et al. [QCD-TARO Collaboration], Nucl. Phys. B 577, 263 (2000) [arXiv:hep-lat/9911033].

[3] M. Creutz, A. Gocksch, M. Ogilvie and M. Okawa, Phys. Rev. Lett. 53, 875 (1984).

[4] M. Creutz, Phys. Rev. Lett. 50, 1411 (1983).

[5] E. T. Tomboulis, PoS LAT2005, 311 (2006) [arXiv:hep-lat/0509116].

[6] R. H. Swendsen, Phys. Rev. Lett. 47, 1775 (1981).

[7] T. A. DeGrand, A. Hasenfratz, P. Hasenfratz, F. Niedermayer and U. Wiese, Nucl. Phys. Proc. Suppl. 42, 67 (1995) [arXiv:hep-lat/9412058].

[8] T. Takaishi, Mod. Phys. Lett. A 10, 503 (1995).

[9] M. Hasenbusch and S. Necco, JHEP 0408, 005 (2004) [arXiv:hep-lat/0405012].

[10] A. Bazavov, B. A. Berg and U. M. Heller, Phys. Rev. D 72, 117501 (2005) [arXiv:hep-lat/0510108].

[11] M. Hasenbusch, K. Pinn and C. Wieczerkowski, Phys. Lett. B 338, 308 (1994) [arXiv:hep-lat/9406019]. 